\title{
A 4-MANIFOLD WHICH ADMITS NO SPINE
}

\author{
BY YUKIO MATSUMOTO ${ }^{1}$
}

Communicated by Edgar Brown, Jr., October 26, 1974

1. This note is to present a new example which reveals the impossibility of embedding a 2-torus in a 4-manifold.

THEOREM 1. There exists a compact 4-dimensional PL manifold $W^{4}$ with boundary satisfying the following conditions: (i) $W^{4}$ is homotopically equivalent to the 2-torus $T^{2}=S^{1} \times S^{1}$, and (ii) no homotopy equivalence $T^{2} \rightarrow W^{4}$ is homotopic to a PL embedding.

By a PL embedding is meant one which is not necessarily locally flat.

Theorem 1 is an application of the codimension two surgery theory developed in our previous papers [4] , [5], [6]. The phenomena of "total spinelessness" in higher dimensions (with finite $\pi_{1}$ 's) were found by Cappell and Shaneson [2] using another method of surgery ${ }^{2}$ [1].

A calculation in our proof leads to another consequence concerned with submanifolds in codimension two. Let $K^{4 n}$ denote a product $\mathrm{CP}_{2} \times \cdots \times$ $\mathrm{CP}_{2}$ of $n$-copies of the complex projective plane $\mathrm{CP}_{2}$.

THEOREM 2. For each $n \geqslant 0$, there exists a locally flat embedding $h_{(4 n)}$ of $K^{4 n} \times S^{1}$ into the interior of $K^{4 n} \times D^{2} \times S^{1}$, which is homotopic to the zero cross section $K^{4 n} \times\{0\} \times S^{1}$, but is not locally flatly concordant to a splitted embedding.

A splitted embedding (with respect to a point $*$ of $S^{1}$ ) means a locally flat embedding $f: K^{4 n} \times S^{1} \rightarrow K^{4 n} \times D^{2} \times S^{1}$ such that (i) $f$ is transverse regular to $K^{4 n} \times D^{2} \times\{*\}$ so that the intersection $M^{4 n}=f\left(K^{4 n} \times S^{1}\right) \cap$ $K^{4 n} \times D^{2} \times\{*\}$ is a closed manifold, and (ii) the inclusion $M^{4 n} \rightarrow K^{4 n} \times$ $D^{2} \times\{*\}$ is a homotopy equivalence.

Theorem 2 contrasts with Farrell and Hsiang's result [3] which may be

AMS (MOS) subject classifications (1970). Primary 55C45, 57C65, 18F25; Secondary 55A25, $55 \mathrm{C} 35$.

1 The author is partially supported by the Füjukai Foundation.

2 Their theory (with $\Gamma$-groups) and ours (with $P$-groups) are not the same but both admit a more general unifying algebraic treatment [7]. 
considered as the splitting theorem in codimension $\geqslant 3$.

2. Construction of $W^{4}$. Let $h: S^{1} \rightarrow S^{1} \times D^{2}$ be an embedding indicated in Figure 1. Essentially the same embedding $S^{1} \rightarrow S^{1} \times S^{2}$ was used by Mazur [8] to construct a contractible 4-manifold.

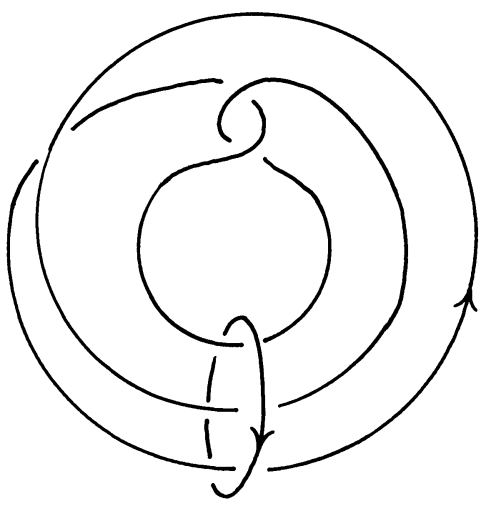

FIgURE 1. Mazur's embedding

Extend $h$ to a framed embedding $\bar{h}: S^{1} \times D^{2} \rightarrow S^{1} \times D^{2}$ in such a way that $\bar{h}$ followed by the natural inclusion $S^{1} \times D^{2} \rightarrow S^{3}$ is isotopic to a trivial knot with a trivial framing. Our manifold $W^{4}$ is the mapping torus of the framed embedding $\bar{h}$. More precisely, $W^{4}$ is obtained from a product $S^{1} \times D^{2} \times$ $[0,1]$ by identifying $(x, \xi) \times\{1\}$ with $\bar{h}(x, \xi) \times\{0\}$ for each $(x, \xi) \in S^{1} \times$ $D^{2}$. Since $h$ is homotopic to the zero cross section $S^{1} \times\{0\} \rightarrow S^{1} \times D^{2}$, $W^{4}$ is homotopically equivalent to $T^{2}$.

Moreover, the embedding $h_{(4 n)}$ in Theorem 2 is nothing other than $\operatorname{id}_{K} \times h: K^{4 n} \times S^{1} \rightarrow K^{4 n} \times S^{1} \times D^{2}, h$ being Mazur's one.

3. Sketch of proof. We first give some generalities. Suppose a compact connected oriented PL $2 n+2$-manifold $V^{2 n+2}$ has the same simple homotopy type as an oriented Poincaré complex of formal dimension $2 n \geqslant 6$. Let $\pi \rightarrow$ $\pi^{\prime}$ denote the associated (onto) homomorphism with $V^{2 n+2}$ defined to be $\pi_{1}(V-L) \rightarrow \pi_{1}(V)$, where $L^{2 n}$ is an exterior $n$-connected (i.e. taut) $2 n$-submanifold of $V^{2 n+2}$ [4]. The kernel of $\pi \rightarrow \pi^{\prime}$ is generated by a (specified) central element $t$ represented by a fiber of the associated $S^{1}$-bundle with a 2-disk bundle neighbourhood $N$ of $L^{2 n}$.

A $(-1)^{n}$-Seifert form over $\pi \rightarrow \pi^{\prime}$ is, by. definition, a (not necessarily nonsingular) $(-1)^{n} t$-Hermitian form defined over $Z \pi$ which becomes nonsingular over $\mathbf{Z} \boldsymbol{\pi}^{\prime}$ (after tensored with $\mathbf{Z} \boldsymbol{\pi}^{\prime}$ ). 
Then the left $\mathbf{Z} \pi$-module $\pi_{n+1}(V-L, N-L)$ is proved to carry a $(-1)^{n}$-Seifert form whose class in $P_{2 n}\left(\pi \rightarrow \pi^{\prime}\right)^{3}$, the "Witt group" of $(-1)^{n}$. Seifert forms over $\pi \longrightarrow \pi^{\prime}$, does not depend on $L$. Denote the class by $\eta(V) \in P_{2 n}\left(\pi \rightarrow \pi^{\prime}\right)$. Then $\eta(V)=0$ if and only if $V$ admits a locally flat spine [6].

Now with the notations of $\S 2$, the product $W^{4} \times \mathrm{CP}_{2}$ has the homotopy type of $T^{2} \times \mathbf{C P}_{2}$. The associated homomorphism with it is $\{\mathrm{Z} \times \mathrm{Z} \times$ $\mathbf{Z} \rightarrow \mathbf{Z} \times \mathbf{Z}\}=(\mathbf{Z} \rightarrow 1) \times \mathbf{Z} \times \mathbf{Z}$, and the obstruction element $\eta\left(W^{4} \times \mathbf{C P}_{2}\right)$ is proven to be in the image of the injective homomorphism

$$
j_{*}: P_{6}((\mathrm{Z} \longrightarrow 1) \times \mathrm{Z}) \rightarrow P_{6}((\mathrm{Z} \rightarrow 1) \times \mathrm{Z} \times \mathrm{Z}) .
$$

Let $\eta^{\prime}=j_{*}^{-1}\left(\eta\left(W^{4} \times \mathrm{CP}_{2}\right)\right)$.

LEMMA 1. The element $\eta^{\prime}$ of $P_{6}((\mathrm{Z} \rightarrow 1) \times \mathrm{Z})$ is represented by $a$ (-1)-Seifert form $(G, \lambda, \mu)$ given by: $G=\Lambda x_{1} \oplus \Lambda x_{2}, \lambda\left(x_{1}, x_{2}\right)=-s^{-1}$, $\mu\left(x_{1}\right)=s-1, \mu\left(x_{2}\right)=-1$, where $\Lambda=\mathrm{Z}\left[t, t^{-1}, s, s^{-1}\right], t$ (or s) denoting the positive generator of the first (or the second) $\mathrm{Z}$ of $(\mathrm{Z} \rightarrow 1) \times \mathrm{Z}$.

REMARK. The matrix $\left(\lambda\left(x_{i}, x_{j}\right)\right)$ of the $(-1)$-Seifert form of Lemma 1 is

$$
\left(\begin{array}{cc}
(s-1)-\left(s^{-1}-1\right) t, & -s^{-1} \\
s t, & -1+t
\end{array}\right),
$$

the determinant of which coincides (up to units) with the Alexander polynomial of Mazur's link (Figure 1) calculated by the method of Torres and Fox [9].

LEMMA 2. $\eta^{\prime}$ is not in the image of

$$
i_{*}: P_{6}(\mathrm{Z} \rightarrow 1) \rightarrow P_{6}((\mathrm{Z} \rightarrow 1) \times \mathrm{Z}) .
$$

The proof of Theorem 1 goes as follows. Suppose that there were a spine $T_{0}^{2} \subset W^{4}$. $T_{0}^{2}$ may be assumed to be locally flat except at one point. The product $T_{0}^{2} \times \mathrm{CP}_{2}$ is a spine of $W^{4} \times \mathrm{CP}_{2}$ whose singularity is of the type (knot cone) $\times \mathbf{C P}$. Since $\pi_{1}\left(\{p t\} \times \mathbf{C P} P_{2}\right)=\{1\}$, this singularity is replaced by a knot cone singularity over a knotted 5-sphere in a 7-sphere [4], $[6, \S 6.4]$. This implies that the $\eta\left(W^{4} \times \mathrm{CP}_{2}\right)$ is in the image of $j_{*} \circ i_{*}$, since $P_{6}(Z \rightarrow 1)$ is isomorphic to the $(7,5)$-knot cobordism group [6]. However, this contradicts Lemma 2.

${ }^{3}$ This notation slightly differs from the original one [6]. 
REMARK. If we start the construction with the embedding indicated in Figure 2, we will obtain $W^{4^{\prime}}$ which admits a locally flat spine.

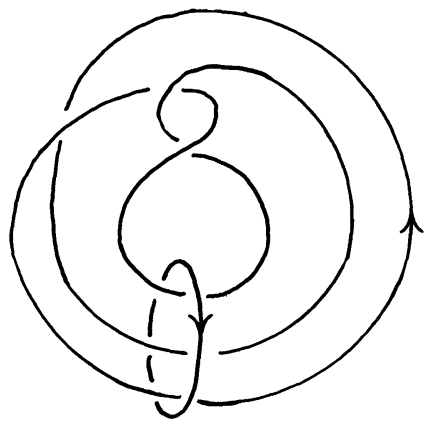

FIGURE 2. False embedding REFERENCES

1. S. E. Cappell and J. L. Shaneson, The codimension two placement problem and homology equivalent manifolds, Ann. of Math. (2) 99 (1974), 277-348.

2. - Totally spineless manifolds, Topology (to appear).

3. F. T. Farrell and W. C. Hsiang, A geometric interpretation of the Künneth formula for algebraic K-theory, Bull. Amer. Math. Soc. 74 (1968), 548-553. MR 37 \#274.

4. M. Kato and Y. Matsumoto, Simply connected surgery of submanifolds in codimension two. I, J. Math. Soc. Japan 24 (1972), 586-608. MR 46 \#6369.

5. Y. Matsumoto, Surgery and singularities in codimension two, Proc. Japan Acad. 47 (1971), 153-156. MR 45 \#6008.

6. Knot cobordism groups and surgery in codimension two, J. Fac. Sci. Univ. Tokyo, Sect. IA 20 (1973), 253-317.

7. Some relative notions in the theory of Hermitian forms, Proc. Japan Acad. 49 (1973), 583-587.

8. B. Mazur, A note on some contractible 4-manifolds, Ann. of Math. (2) 73 (1961), 221-228. MR 23 \#A2873.

9. G. Torres and R. H. Fox, Dual presentation of the group of a knot, Ann. of Math. (2) 59 (1954), 211-218. MR 15, 979.

DEPARTMENT OF MATHEMATICS, COLLEGE OF GENERAL EDUCATION, UNIVERSITY OF TOKYO, KOMABA, MEGUROKU, TOKYO, 153, JAPAN 